\title{
Los encuentros lúdicos
}

\section{Su origen,}

\section{necesidades $y$}

filosofia

FARID SALGADO CAJALES*

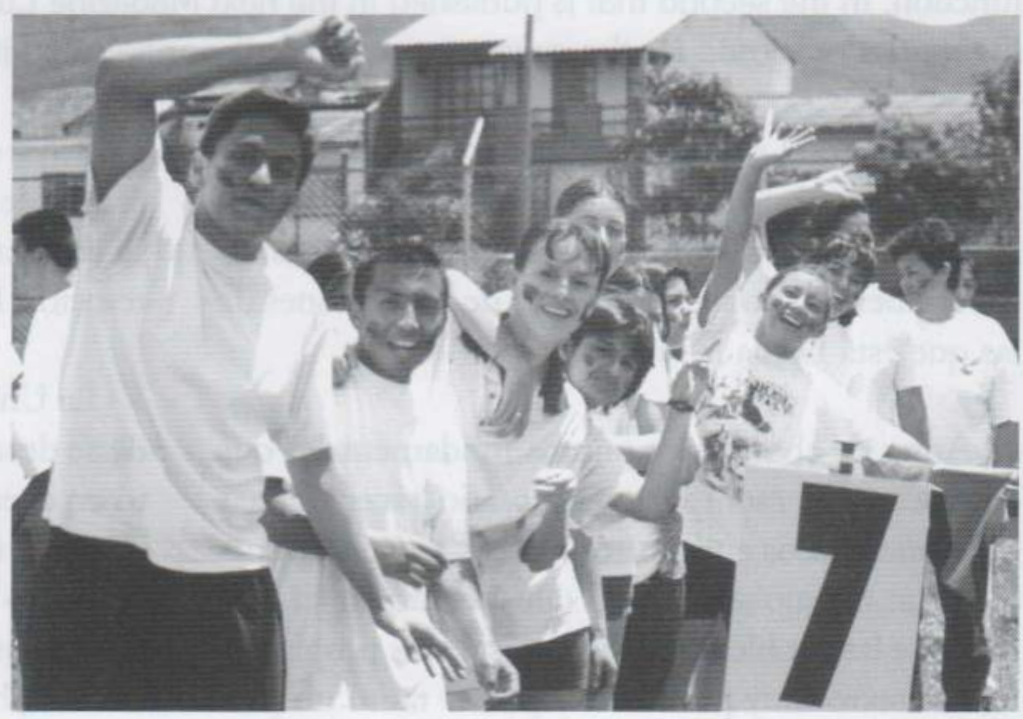

Por: Ricardo Neme Barrero

\section{Resumen}

\section{T}

Jos encuentros lúdicos son eventos que surgen a partir de las características y necesidades que presenta la Unión de Colegios Bilingües (UCB). Podrían ser definidos como una situación construida de encuentro con el otro, fundamentada en el juego y la dimensión lúdica de lo humano, que busca la exploración y constitución de sentido propio a través de la creación individual y colectiva, y el establecimiento de unas formas de relación y proceder que favorezcan la convivencia. Esta es la primera parte de dos: en la primera se esbozan las condiciones y características del contexto que dieron paso al planteamiento del proyecto, definiendo los principales conceptos que involucran el encuentro tanto en su estructura como en su función. En la segunda, que se publica en la próxima Revista Lúdica, se describen y explican los juegos propuestos para el encuentro y la disposición que se espera lograr desde su dinámica.

Fecha de recepción: octubre 10 de 2003 - Fecha de aceptación: octubre 23 de 2003

Palabras clave: encuentro con el otro, situación construida, comportamiento experimental, participación efectiva, multipolar.

\section{Abstract}

The encounters lúdicos are events that arise starting from the characteristics and necessities that it presents the Union of Bilingual Colleges (UCB). They could be defined as a built situation of encounter with the other one, based in the game and the dimension lúdica of the human thing that it looks for the exploration

\footnotetext{
* Profesor Departamento de Educación Física, Universidad Libre. Bogotá
} 
and constitution of own sense through the individual and collective creation, and the establishment in some relationship ways and proceed that they support the coexistence. This is the first part of two: in the first one the conditions and characteristics of the context are sketched that make away for the position of the project, defining the main concepts that involve the encounter as much in their structure as in their function. In the second that is published in the next Magazine Lúdica, they are described and they explain the games proposed for the encounter and the disposition that it hoped to achieve from their dynamics.

"El juego-el que aún no ha sido pervertido por el mortal y aburrido fanatismo competitivo, que arruina todo placer para conseguir un record estulto- es libertad, ironía, conciencia de las ficciones de las que está hecha la vida"

Antes de abordar los puntos fundamentales que convocan este escrito, creo necesario hacer algunas sugerencias. Los encuentros lúdicos son el producto de un trabajo académico que atendió en su momento a las problemáticas y necesidades propias de un contexto muy particular y por lo tanto no pretende que sea asumido desde la arrogante categoría de universalidad. Esta propuesta no tiene el interés de ser apropiada como recetario, ni asegura resultados definitivos, por respeto a la diversidad de los factores que determinan un contexto y a la lectura particular que cada grupo humano hace de sus necesidades. Así, los encuentros lúdicos los entendemos como una propuesta pertinente considerada desde un punto de vista particular y restringido.

Quizá lo único rescatable en términos de universalidad sea su proceso de constitución: reconocer unas necesidades, atender a la problemática que genera la satisfacción de tales necesidades, estudiar las respuestas que se encuentran en marcha de cara a la favorabilidad o desfavorabilidad de las soluciones frente a un proyecto de desarrollo humano, y de no encontrar satisfacción en las soluciones vigentes, diseñar una propuesta alternativa que aborde lo que es considerado sustancial por el grupo humano que es causado y causante de dicho contexto, atendiendo en todo momento a un elemento que es fundamental en todo proceso de convivencia pacífica, la argumentación.

Cualquiera de los elementos, conceptos o experiencias que se van a presentar a continuación y puedan ser objeto de su interés, se recomienda sea sometido a una prueba de favorabilidad de cara a las particularidades de su contexto. Es un ejercicio sano de reconocimiento y respeto a la diversidad de lo humano.

La Unión de Colegios Bilingües (UCB), en donde se desarrolla la propuesta, nació y se fundamentó en el encuentro con el otro, un encuentro intencionado desde un proyecto educativo común. El conjunto de actividades: académicas, lúdicas, artísticas, musicales y deportivas, que convocan al encuentro, han sido orientadas desde el reconocimiento y respeto por las diferencias, la democratización de la participación y el establecimiento de vínculos que generen reconocimiento social y sentido de pertenencia. Situarse en la diferencia a partir de los múltiples referentes que se generan desde el encuentro e integrarse en el complejo de relaciones que implica la vida en sociedad, es una respuesta que desde la organización se plantea frente a las condiciones de convivencia que caracterizan nuestro país.

Trabajar a la medida de las necesidades de la población escolar en términos del desarrollo, ha sido la tarea que se ha trazado un grupo de licenciados en educación física. Desde las características que presentaba la infraestructura de escenarios propios para la educación física, la recreación y el deporte, se generó la necesidad de una intervención y adecuación a las exigencias de la población escolar en cada nivel: preescolar, primaria, media y bachillerato. Paralelo a la discusión sobre la pertinencia de los escenario se estableció un debate sobre el sentido y las características de las actividades que estaban atravesando los encuentros y se emprendió un proceso de cambio

\footnotetext{
1. Una gran amiga ha enviado, en buena hora, este extracto de Claudio Magris: "la escuela: risa y libertad" del su libro Utopia y desencanto. (2001). Anagrama, p. 312.
} 
enfocado en la vivencia cotidiana de los fundamentos que de dieron origen a la UCB.

Para nadie es un secreto que el deporte es hoy en día objeto de múltiples miradas que intentan dar cuenta de la manifestación real de su discurso. Una vez agotado el discurso biológico-funcionalista, las ciencias humanas se han acercado al deporte para dar cuenta de sus postulados en términos de la construcción de sociedad y desarrollo humano.

Atendiendo algunas reflexiones sobre los valores agregados y los aspectos intrínsecos en la estructura y función del deporte como espectáculo y rendimiento, optamos por construir situaciones de encuentro igualmente lúdicas que contrarrestaran aquellos elementos de dicho modelo, que desde nuestra mirada no resultan favorables para el desarrollo de los niños entre los 7 y 9 años; esperando que las disposiciones generadas desde los encuentros lúdicos, nos permitan en el mediano plazo establecer una diferenciación real entre el deporte espectáculo y el deporte escolar.

Algunos de los elementos que se han intentado neutralizar a partir de cambios estructurales y de sentido en los encuentros lúdicos son: la relación del deporte con el establecimiento de disposiciones a la dominación, la interacción con el adversario orientada exclusivamente desde el conflicto y la rivalidad, los mecanismo de determinación social generados desde la selección y el resultado, las disposiciones del espacio, el tiempo y el error como elementos sancionatorios, la competencia como elemento de ruptura en la relaciones humanas, la tecnificación de la expresión corporal y el proceso de pedagogización que han tenido los excesos de las manifestaciones del hombre dentro del escenario deportivo.

La premisa de este proyecto es que queremos un cambio en las condiciones de vida que caracteriza los modos de relación en el contexto colombiano; pretendemos superar deliberadamente los modelos de relación establecidos en el marco del deporte espectáculo. Queremos una sociedad más tolerante frente a la opción del otro, queremos que exista la posibilidad de un otro, queremos espacios en donde se encuentren las diferencias, modos y sentidos de vida diversos, sin que ello implique caer en lo vulgar. Pretendemos construir situaciones ${ }^{2}$ que derrumben las barreras artificiales e intervengan las rupturas generadas en las relaciones humanas. Es por ello que este proyecto halló su sentido en la denominación "encuentro" y se define así mismo como una situación construida de encuentro, alimentada desde la dimensión lúdica de lo humano y que atiende a un proyecto educativo interinsti-tucional. Los encuentros lúdicos pretenden ser el producto $\mathrm{y}$ a su vez el instrumento de nuevos comportamientos, nuevas formas de relacionarnos. Esperamos en unos años poder dar cuenta de una lúdica en el establecimiento de las relaciones humanas.

Para ello se ha intervenido el espacio, el escenario del encuentro, de tal manera que disponga de una experiencia de comportamiento alternativo frente al adversario y que deliberadamente invite a la organización colectiva. Así, la creación de ambientes diversos, con lógicas de expresión y manipulación de los elementos diferenciadas, y la rotación por todos los ambientes, contribuye a la configuración de un comportamiento experimental en cada uno de los participantes. La ausencia de verdades pre-establecidas, estándares o prescripciones, en lo técnico y en lo táctico, potencializa el valor del ensayo, del proceso, de la participación directa y de la "equivocación" como plataforma de desarrollo y abre paso a la manifestación de tendencias y estilos.

Quizá una de las puertas de salida al conflicto permanente que tenemos los colombianos en el tema de la convivencia, sea la de encontrar sentido al interior de nosotros mismos, de nuestras manifestaciones culturales, de nuestro espacio. Lo que se pretende al generar las condiciones para el surgimiento de un comportamiento experimental es precisamente la construcción de sentido desde lo vivi-

2. Entre los muchos padres que tiene los encuentros lúdicos, como ejercicio académico, debo destacar a Guy Debord y la internacional situacionista. 
do e intervenido directamente. Cuando aflora el estilo se puede decir que hemos ganado una batalla contra la alineación, cuando cada niño comienza a encontrar sus propias maneras de solucionar los problemas que le plantean los ambientes, respetando los acuerdos alcanzados colectivamente, se puede decir que está construyendo sentido en sí mismo. Al finalizar los encuentros un niño puede encontrar en su forma de pasar, en su forma de evadir, en su forma de patear, algo que realmente le pertenece, que no fue impreso en su cuerpo desde alguna ortodoxia.

En este marco conceptual resulta poco favorable la figura del entrenador o la del director técnico desde la lógica que se expone en los torneos intercolegiados o la del deporte espectáculo. Además, para los encuentros lúdicos no se entrena, se juega y ese juego no pretende ser contabilizado ni medido. Ese momento del encuentro es para expresar y manifestar el mundo interior, y para sembrar la semilla de la autarquía. Se trata de crear una lógica colectiva a partir de la participación directa y ese proceso, propio del juego, no hace necesaria la figura del entrenador. El rol del adulto se limita a la mediación de conflictos siempre y cuando sea convocado por los niños para ello o cuando la situación lo amerita.

Resaltar la búsqueda de sentido en sí mismo no implica negarnos a explorar sentidos que se encuentran fuera de nosotros. Puede ser tan desfavorable encerrarnos en nuestro propio mundito como el dejarnos conducir exclusivamente por una cultura prestada. Emprender la búsqueda o construcción de algo que nos pertenezca de veras, puede ser un elemento catalizador de las condiciones de existencia que caracterizan nuestra sociedad y especialmente al enclasamiento social en donde se desarrolla la propuesta.

Desde las implicaciones del encontrar sentido en sí mismo es que nos hemos negado a las premiaciones selectivas, al establecimiento de jerarquías y al empoderamiento del resultado como estigma de las relaciones. La razón de ser del encuen- tro puede hallarse en el encuentro mismo, en el goce percibido, en lo vivido, en el comportamiento que se experimenta. No importa identificar quién fue el primero o el último, no existen razones o motivos para ello, cada cual se lleva en sí mismo las ganancias del encuentro. No se quiere generar la utopía del campeón que muchas veces domina el hecho y la actividad misma del encuentro reduciéndola al resultado y negando el proceso sentido por todos aquellos que participaron del encuentro. $\mathrm{Ni}$ desde el resultado, ni desde los premios, ni desde categorías de eficiencia y eficacia se pretende encontrar sentido o explicación a los encuentros lúdicos. Partimos del deseo de jugar e intentamos que no se distorsione demasiado ese deseo auténtico. Al finalizar el año escolar, en el último encuentro del año, se propone una copa de helado y un "recordatorio" para todos los participantes, en igualdad de condiciones. Para ese evento, esperamos se hayan diluido las identidades institucionales y que todos los participantes, de manera espontánea, compartan ese momento desde los nuevos vínculos establecidos.

Como el deseo de jugar es propio de la condición humana, hemos logrado superar la etapa de las selecciones o elección de los mejores. La invitación a los encuentros se realiza por grados o por cursos, no por selecciones. No importa el número, ni el género; lo importante es que todos los niños pertenecientes a una institución escolar en el grado o nivel en que se hace la convocatoria hagan uso de su derecho a jugar. Educarnos en ese derecho, sin importar talla, peso o cuanto mecanismo de selección se invoque, es fundamental en la ruptura de la lógica de los encuentros intercolegiados y cotidianidad escolar. Lograr una cobertura total de la población escolar en esas edades, con una participación efectiva, es una de las prioridades del proyecto.

La participación efectiva, que entendemos como una manifestación protagónica del niño en todas las fases de cada juego, se ha logrado a partir del control del número de participantes por juego y la simultaneidad en la oferta de problemas durante cada juego. Con el control del número de participantes se pretende que no se acceda a la delegación 
de la responsabilidad que implica participar y así, negar la posibilidad de definir funciones especializadas permanentes durante el juego, de tal manera que cada niño logre inmiscuirse en la totalidad de las fases de cada juego.

La oferta simultánea de diferentes problemas en cada juego, es uno de los elementos estructurales que más disponen a la participación efectiva. La tendencia en los deportes tradicionales es la atomización de la atención en un solo elemento y la dinámica se polariza en fases que se presentan de manera alterna -primero la defensa y luego el ataque o viceversa-, lo que posibilita procesos de monopolización del elemento o la situaciones. $\mathrm{Al}$ ofrecer más de una sola pelota, más de una sola meta, más que una relación univoca de adversidad, hemos generado una multipolaridad en los juegos y multiplicado las responsabilidades y posibilidades de protagonizar acciones. Es posible que en un mismo momento un equipo este viviendo simultáneamente dos fases del juego; ataque y defensa, o tenga que estar defendiendo su meta mientras colabora en la defensa de la meta de otro equipo. Esta descentralización de los problemas del juego, dificulta los procesos de monopolización de los elementos y convoca a cada niño a una intervención directa en todas las fases del juego. El espíritu de la participación efectiva es democratizar las posibilidades de éxito en el marco de una construcción y producción colectiva.
Uno de los elementos vitales en la configuración de un contexto democrático es la participación; pero la participación deviene acción y la acción deviene interacción. Que las ideas antecedan o precedan la acción, no es un tema de debate en este documento, lo importante es que surjan las ideas y en especial aquellas que puedan afectar las condiciones de existencia que no favorecen nuestra convivencia. Los niños en el marco de este proyecto tienen la posibilidad de intervenir todas las esferas del juego; desde la normatividad en adelante esta dispuesta a ser modificada por ellos. Lo fundamental es que a partir de su acción e interacción perciban que esos frutos les pertenecen, que al ser los protagonistas del proceso también son los beneficiados por el resultado, o en otras palabras, que ellos son, o mejor, vuelven a ser, productores y consumidores de sus propias emociones.

Es importante destacar que con la extensión de la jornada escolar, el juego auténtico y espontáneo fue reemplazado por las escuelas deportivas y unas horas más de español y matemáticas. De ahí, que los encuentros lúdicos se hallan incrustado en el centro mismo de la cotidianidad escolar, para agitarla, para ocasionar una ruptura en su monotonía, para disminuir su tiempo muerto. Los juegos propuestos pretenden encender la emoción en medio de ese desierto de pasiones e intuiciones en que ha sido convertida la vida escolar. 